
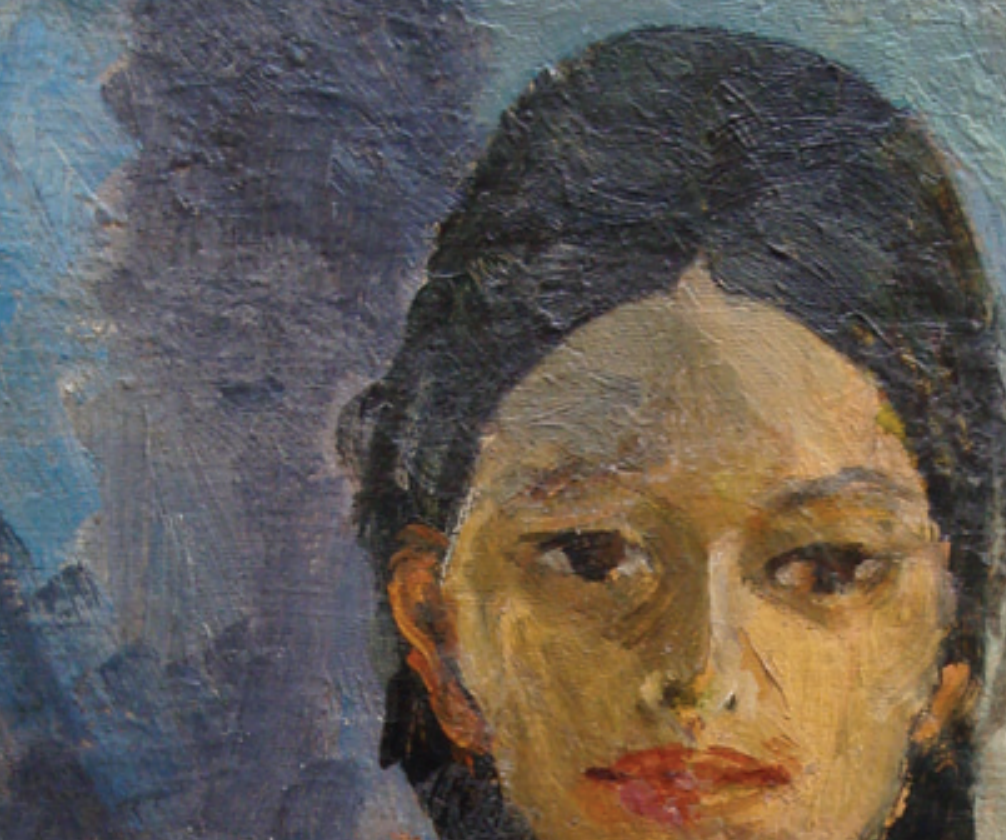

Ne

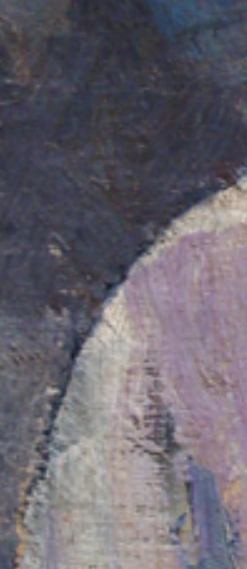

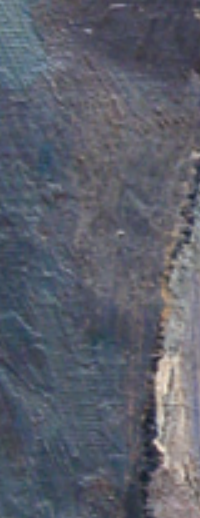
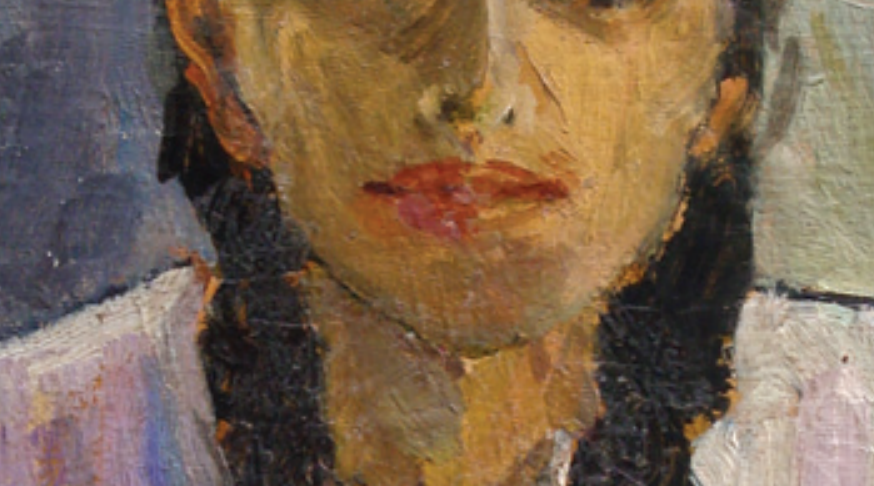
I. $\left.(4)^{4}\right) e^{2}$ tid $y=1 \frac{1}{5}$

हhe

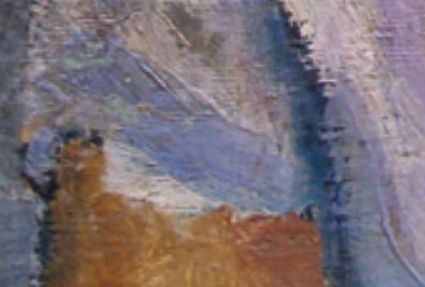

at)
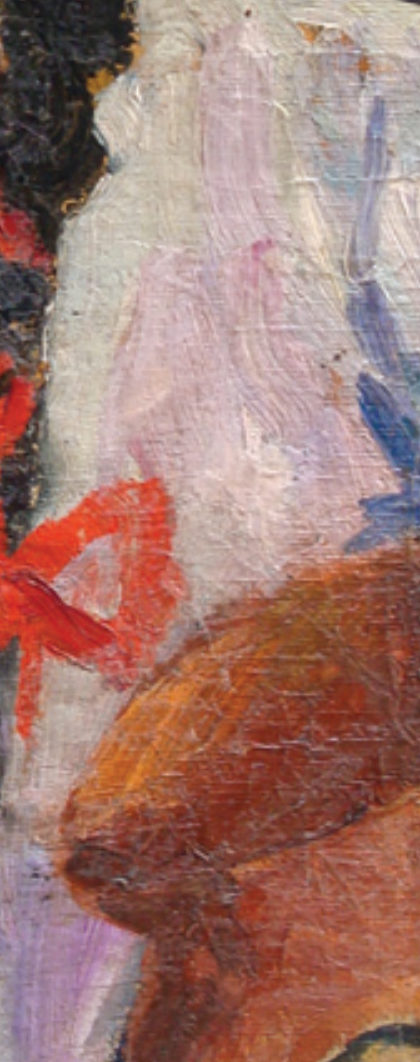

2. 1 (2)

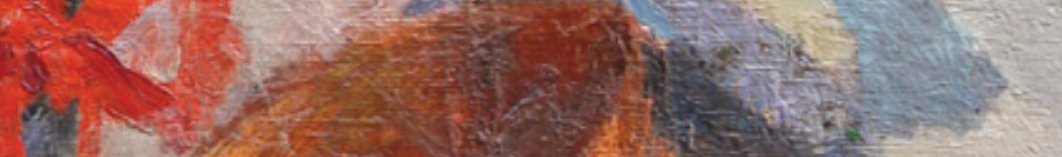

is $\rightarrow$
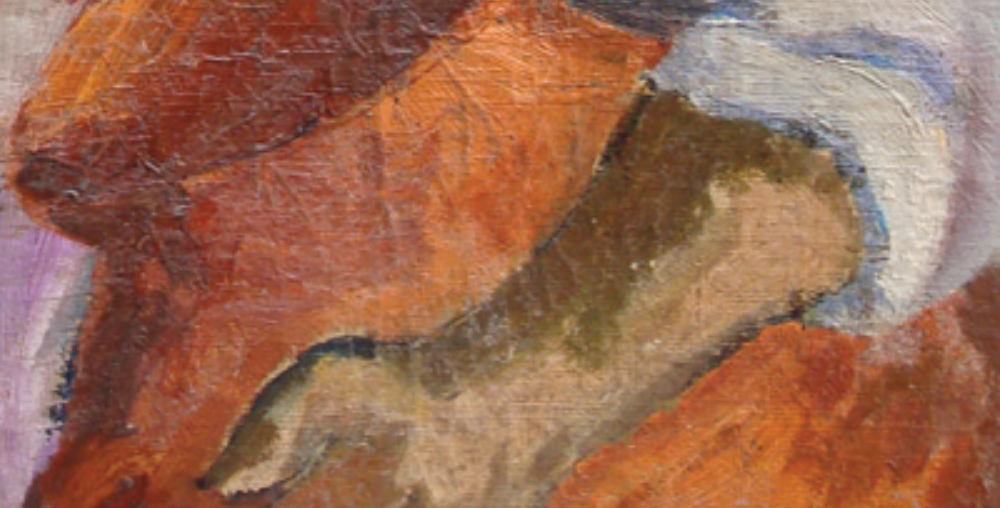


\section{Situación del acceso al servicio de agua potable de la población del municipio de Intibucá}

Edith Yolany Zelaya

\section{INTRODUCCIÓN}

Una de las metas del milenio es "Reducir a la mitad, para el año 2015, el porcentaje de personas que carecen de acceso sostenible a agua potable", la importancia de este objetivo radica en que el agua es una necesidad básica del ser humano.

Los seres vivos tenemos dependencia de agua durante toda nuestra existencia, los humanos la necesitamos para consumir, para producir, para negociar es una necesidad básica para sobrevivir en el planeta.

Aunque Honduras cuenta con suficientes fuentes de agua para toda la población, esta no es accesible para todos. Existen muchas desigualdades en relación al acceso al agua especialmente cuando se trata de agua potable. Las poblaciones de las áreas rurales siguen siendo las más afectadas.

La falta de agua, consumir agua contaminada, condiciones de vida sin higiene tienen como principales consecuencias: salud quebrantada, extrema pobreza y muerte. Simplemente el agua significa VIDA.

Conocer la situación del acceso al agua potable en las comunidades de nuestro país tendría que ser la información básica para tomar decisiones e invertir para combatir la pobreza. La mayor cantidad de personas o de viviendas sin acceso a agua potable se ubica en el área rural, es en esta población donde se deben centrar los esfuerzos de erradicación de pobreza; si se tiene agua, se puede producir y por ende comer, dormir, vestirse y educarse.

Se espera que el lector o lectora de esta investigación conozca en números cuál es la situación con respecto al acceso de agua potable de las comunidades urbanas y rurales del municipio de Intibucá; y si usted tiene la oportunidad de tomar decisiones para beneficio de la población intibucana utilice estos resultados y esa será la mayor satisfacción que podré recibir como investigadora.

\section{JUSTIFICACIÓN}

En el planeta tierra los seres humanos somos los actores principales del manejo de los recursos naturales, el recurso agua es el elemento esencial para la vida humana. La supervivencia depende de tener o no agua para consumir y para producción de alimentos y estos a su vez para mejorar economías. Se estima que un $80 \%$ de las enfermedades en la población más vulnerable son causadas por el consumo de agua contaminada. He ahí la importancia de que cada hondureño y hondureña cuente con el acceso a un servicio de agua potable.

La población hondureña con acceso a agua potable es de un $70 \%$ aproximadamente, la falta de agua tiene mucho que ver con la pobreza ya que afecta a sectores como salud reflejándose en diarreas y desnutrición; en el sector educación hay ausentismo en las aulas y en relación al ingreso familiar la canasta básica se encarece más si hay que comprar el agua y luego se refleja en una mala alimentación.

La investigación sobre la situación del acceso a agua potable en las viviendas del municipio de Intibucá tiene como propósito fundamental conocer cifras confiables derivadas del Censo de Población y Vivienda del 2001 
que nos demuestren la realidad de una de las necesidades básicas del ser humano. La información servirá como un punto de partida para tomar decisiones puntuales de inversión dentro del municipio y así lograr desarrollo y avances significativos en las condiciones de vida de las familias intibucanas.

\section{PLANTEAMIENTO DEL PROBLEMA}

El agua es un derecho humano, se dice que Intibucá cuenta con el recurso hídrico pero no se ha canalizado correctamente en el sistema de acueducto para abastecer con mayor eficiencia a la población del municipio.

Actualmente en muchos barrios y colonias del municipio de Intibucá, el servicio de agua potable no es continuo, es un servicio por horas con frecuencia de 10 de la noche a 5 de la mañana; cabe mencionar que en época de invierno las características del agua no son muy agradables ya que es un agua turbia de color café claro. Esta problemática requiere de investigación para conocer la situación actual del servicio de agua potable, y de ser posible conocer las razones por las que el servicio en muchas localidades del municipio no es eficiente.

\section{OBJETIVOS}

\subsection{Objetivo general:}

Conocer la situación del acceso al servicio de agua potable de la población del municipio de Intibucá.

\subsection{Objetivos específicos:}

- Identificar el número de viviendas que tienen el acceso al servicio de agua potable según dominio en el municipio de Intibucá.

- Identificar la procedencia del recurso agua de los diferentes sistemas de agua potable con que cuenta la población del municipio de Intibucá.

\section{METODOLOGÍA}

El proceso metodológico para la investigación comprende las fases de una revisión bibliográfica e informa- ción que se encuentra en la base de datos del Censo de Población y Vivienda del 2001. Las fases de este proceso son las siguientes:

- Recopilación de información, para ello se procedió a la seleccionar toda la información factible respecto al tema, se accedió a diferentes tipos de fuentes como son: base de datos del CPV 2001; documentos como Anuario Estadístico del 2006; documentación recolectada en Internet : Agua Potable y Saneamiento en Honduras, Derecho Humano al agua Potable, Los servicios de Agua Potable y Saneamiento en el Umbral del XXI; Páginas Web: Programa de las Naciones Unidas para el Desarrollo, Informes del PNUD; Entrevistas con personal del SANAA.

- Sistematización de la Información. Se revisó la documentación recolectada para sintetizar información sobre el consumo de agua potable desagregando desde el nivel mundial hasta el nivel de municipio. Se utilizó el Programa REDATAM y la base de datos del Censo Poblacional y Vivienda del 2001, de la base se extrajo información cuantificable del departamento de Intibucá, del municipio, aldeas y caseríos. La información recolectada de Internet se revisó detalladamente, utilizándose la pertinente.

- Análisis de información. Se analizó, evaluó e interpretó la información, a partir de ello se elaboró el informe, estableciendo luego las conclusiones generales del presente estudio basado en los objetivos propuestos. se utilizó hojas de cálculo de excell que facilitaron la elaboración de cuadros y gráficos.

\section{CONTEXTO DEL ESTUDIO}

\subsection{Contexto geográfico de la investigación}

El departamento de Intibucá se encuentra en la región occidental del país; según Índice Desarrollo Humano es el décimo departamento más pobre, tiene un IDH de 0.582 y un Índice de Pobreza de 37.9, cuenta con 17 municipios y 127 aldeas (PNUD-IDH 2006).

Según el Censo Poblacional del 2001 se registraron 34,716 viviendas que se encuentran localizadas en 20 aldeas y un total de 129 comunidades, el área urbana 
comprende 12 barrios que conforman la aldea de Intibucá (INE 2001).

El municipio de Intibucá presentó un Índice de Desarrollo Humano de 0.619 para el 2004 y el IPH de 34.3; el municipio de Intibucá para el 2008 contaba con una población de 50,855 delos cuales 24,535 eran hombres y 26,320 mujeres. Un 75\% de la población del municipio sabe leer y escribir, se cuenta con centros educativos desde el nivel de pre-básico hasta nivel universitario, así mismo hay oportunidad de educación bilingüe. La actividad agrícola es la principal fuente de ingresos en el municipio.

\subsection{Situación del acceso al servicio de agua potable} de la población del municipio de Intibucá

El agua es un recurso vital para el ser humano y el derecho al agua potable y saneamiento forman parte integral de los derechos humanos. El acceso al agua debe ser considerado como un derecho básico, individual y colectivamente inalienable. Probablemente quien controle el agua controlará la economía y la vida en un futuro no muy lejano.

Desde 1950 se ha triplicado con creces el uso del agua en el mundo durante los últimos 25 años; la disponibilidad del agua en el mundo disminuyó un 50\%. Si se continua esta tendencia se proyecta que para el 2025, 3,500 millones de personas sufrirán problemas con el acceso al agua; así mismo la cantidad de gente que vive en países con estrés por falta de agua pasará de 470 millones a 3,000 millones.

Según los datos del Informe de Desarrollo Humano del 2006 presentado por el Programa de Desarrollo de las Naciones Unidas (PNUD) casi 50 millones de personas no tienen acceso a agua potable en Latinoamérica y el Caribe y unos 119.4 millones de personas no tienen acceso a sistemas de saneamiento. A escala global, la cifra de personas sin acceso al agua potable es de 1,100 millones y la de los que no disponen de sistema de saneamiento como letrinas, es de 2,600 millones.

"Según Guissé H.1997, en el ser humano, la pérdida de agua puede tener consecuencias graves, si alcanza el 10\% de la masa presente en el cuerpo, y provocar la muerte a partir del 20\%. Por otra parte, aunque el agua está siempre cargada de diferentes sustancias minerales y orgánicas, su contenido en el hombre adulto y en buena salud va del 58 al 67\%, mientras que en el recién nacido es del orden del 66 al 74\%".

Se ha comprobado que los deficientes servicios de agua y saneamiento son la causa directa del deterioro de la salud, según la OMS el impacto de la falta de agua segura, se traduce en casi la mitad de los habitantes de los países en desarrollo, sobre todo los niños y niñas son los más afectados por enfermedades causadas por el consumo de aguas y alimentos contaminados provocando que 6,000 niños y niñas mueran cada día en todo el mundo, principalmente por diarreas que se convierte en la segunda causa de muerte infantil.

\subsection{Situación del acceso de agua en América Latina y Honduras}

La problemática de disponibilidad y calidad de agua en América Latina obedece a varios factores según el informe del Banco Mundial sobre la Salud y Medio Ambiente en 2003: "en la mayoría de los casos el problema del agua en la región obedece a la falta de un marco jurídico, institucional y normativo adecuado, a las enormes distorsiones en los precios y a los servicios subsidiados que benefician a los sectores más prósperos de la sociedad en detrimento de los pobres." (www.monografias.com)

Según el Programa de las Naciones Unidas para El Medio Ambiente a nivel de América Latina un 15\% de la población (alrededor de 76 millones de personas), no tiene acceso a agua potable, proporción que se duplica en el caso de las zonas rurales, agregando que un 60\% de las viviendas que cuentan con un sistema de agua no tienen un abastecimiento contínuo (PNUMA, 2003).

En nuestro país, Honduras, según la Encuesta Permanente de Hogares en 2006, el $81 \%$ de las viviendas tenía acceso a agua y un $86 \%$ con acceso a saneamiento. El país tiene un potencial hídrico de $1,542 \mathrm{~m}^{3} / \mathrm{sec}$ pero en el 2006 se usó solamente $88.5 \mathrm{~m}^{3} / \mathrm{sec}$. (6\%), esto incluye $73 \mathrm{~m}^{3} / \mathrm{sec}$ para el riego y $13.14 \mathrm{~m}^{3} / \mathrm{sec}$ para uso doméstico e industrial. El abastecimiento de agua en los sistemas de agua potable proviene principalmente 
de manantiales, ríos y quebradas y de aguas subterráneas. En el área rural se han establecido más de 5,000 sistemas de agua potable los cuales son provenientes en $57 \%$ de manantiales, $34 \%$ de quebradas, $5 \%$ de ríos y un $4 \%$ de aguas subterráneas. (Plataforma de Agua en Honduras. p.13-14)

\section{- El Agua dulce es un recurso esencial para la} salud

El agua es un elemento esencial para la vida humana, para la salud básica y para la supervivencia, así como para la producción de alimentos y para las actividades económicas. Las Naciones Unidas estiman que por aguas contaminadas se producen el $80 \%$ de las enfermedades, la población más vulnerable a estas enfermedades son los niños y niñas de los países en desarrollo. Honduras no es la excepción, según el anuario estadístico del 2006 en el sector salud, a nivel del país se atendieron por diarrea a 195,882 niños registrados en los diferentes hospitales públicos.

\section{¿El agua responsabilidad de quien?}

El agua es responsabilidad de todos los seres que hacemos uso de este recurso, sin embargo, nos preocupamos muchas veces solamente si llega o no agua a la tubería de nuestras casas. El agua es un recurso que no depende solamente de entubarla, es responsabilidad de toda la población habitante de una comunidad, un departamento o un país. Antes de llegar a nuestras casas recorre kilómetros de superficie que puede estar expuesta a actividades de contaminación y acciones que facilitan la disminución de éste vital líquido.

En Honduras contamos con el Servicio Nacional de Acueductos y Alcantarillados (SANAA) y las municipalidades que son las principales autoridades encargadas de velar por el servicio de agua potable. A nivel del departamento de Intibucá se cuenta con una oficina regional del SANAA ubicada en el municipio de Intibucá. El SANAA conjuntamente con la municipalidad y las juntas locales de agua son responsables de que haya accesos al servicio de agua potable en todas las viviendas $(6,258$ según CPV2001) del municipio.

En los barrios o colonias que están los patronatos son los entes en los cuales la municipalidad delega la vigilancia del servicio y en las comunidades la figura más comprometida son las juntas locales de agua. A nivel de comunidades las juntas de agua reciben asistencia técnica de empleados del SANAA quienes supervisan la calidad de agua que llega a las viviendas, así mismo facilitan la coordinación con ONG's presentes en la zona para la ejecución de proyectos de agua para consumo. A pesar de estas buenas relaciones todavía hay viviendas que no cuentan con el servicio de agua potable. Según el control del Sistema de Instalaciones de Acueductos Rurales (SIAR CTOCC, 05-ago-2008) la cobertura del SANAA en el municipio está en un 76.4\%.

\subsection{Situación del acceso de agua en el departa-} mento de Intibucá

En el departamento de Intibucá según las características de 29,596 viviendas en el CPV 2001, un 68.5\% posee el servicio de agua por tubería, la cual procede en un $63.26 \%$ de un sistema público/privado, un $27.02 \%$ de un río o una vertiente, el $4.7 \%$ de aguas subterráneas y un $5 \%$ de otro tipo de procedencia.

A nivel de municipio el comportamiento de procedencia del agua en los sistemas es similar al del departamento. De un total de 6,258 viviendas (CPV 2001) el $73.47 \%$ cuenta con un sistema de agua por tubería y la procedencia del agua está comprendida en un $68.52 \%$ de sistema público/privado, $22.47 \%$ de vertiente o río, $2.63 \%$ de aguas subterráneas y un $6.39 \%$ de otro tipo de procedencia. Sin embargo, el hecho que un $73.47 \%$ de la población del municipio cuente con un sistema de agua por tubería no asegura que sea una servicio de agua potable, puesto que el agua potable es limpia, no contaminada, es agua tratada para el consumo humano. En el área urbana del municipio de Intibucá la municipalidad con la asesoría de la oficina del SANAA son responsables del sistema de agua que de paso lo comparte con el casco urbano del municipio de La Esperanza por ser ciudades gemelas. En el área rural las asociaciones directamente responsables de los sistemas de agua potables son las juntas de agua y los patronatos siempre con asesoría técnica del SANAA. 
La Secretaría de Salud reportó en el 2006 que a nivel del departamento de Intibucá la cifra de niños atendidos por diarrea fue de 11, 647 lo que representa un 5.9\% de la población total atendida, cabe mencionar que se cuentan solamente los que lograron registrarse, indudablemente la población sería mayor si se lograran sumar los casos no registrados. Así mismo se puede asociar el consumo de agua a la desnutrición y las estadísticas del INE en el 2004 presentan una población de desnutrición de 25,238 de los cuales sólo el 58.2\% se reportan en control (Secretaría de Salud 2006).

6.5 Situación del acceso de agua en el municipio de Intibucá

- ¿Cuántas viviendas cuentan con el servicio de agua potable en el municipio de Intibucá?:

Según los datos del Censo Poblacional del 2001 el $68.5 \%(4,288)$ de las viviendas del municipio cuenta con un servicio de agua público o privado, lo que estima que el agua que reciben por lo menos cuenta con un tratamiento en los tanques de captación antes de su consumo. El escenario más preocupante se presenta en un $22.9 \%$ (1,427 viviendas) que obtiene el agua de una vertiente, un río o una laguna, lo que significa que hay riesgos de contaminación y un alto potencial de desarrollo de enfermedades gastrointestinales, se agudiza más si en los hogares no hay conciencia de la importancia del tratamiento de esta agua antes de su consumo. No se puede demeritar las campañas de los centros de salud que regularmente realizan en el área rural para hacer hincapié en la prevención de enfermedades por consumo de aguas contaminadas. En el municipio también están presentes ONG’s que ejecutan acciones para controlar y prevenir esta situación, tal es el caso de SAVE THE CHILDREN, VISION MUNDIAL, PLAN de HONDURAS, CRS (Catholic Relief Services), UNICEF, EDUCCSA, COSEPRADI y USAID; quienes en algunas comunidades han participado en el financiamiento de proyectos de agua potable en coordinación con el SANAA. El gráfico No. 1 presenta la distribución del número de viviendas en cada una de
Gráfico 1: Distribución de Viviendas según

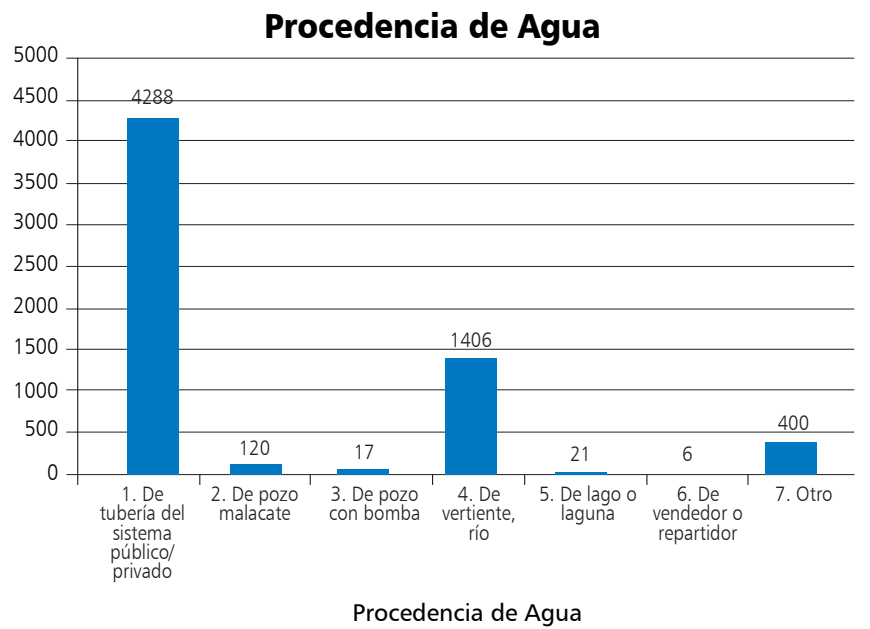

Fuente: Elaboración propia según datos del Censo Poblacional y Vivienda del 2001

las categorías de procedencia del agua según el Censo Poblacional del 2001.

La procedencia de un sistema de tubería público/privado es el que prevalece dentro del municipio cubriendo un porcentaje de $68.5 \%$ de 6,258 viviendas, sin embargo, la procedencia de vertiente o río ocupa el segundo lugar representando un $22.5 \%$ y está sumada a la de lago o laguna más las de pozos se convierte en un número de 1,560 viviendas de cuidado por la contaminación a la que están expuestas las personas al consumir el agua de las procedencias mencionas. En las 400 viviendas de la categoría otros no se sabe con seguridad si cuentan con agua pero por la tendencia podemos decir que se concentran en las dos procedencias más relevantes que son de un sistema o de un río (Cuadro No. 1). Es preciso mencionar que la categoría de otros en los diferentes análisis dentro del censo se refiere a viviendas que no se lograron censar por diferentes circunstancias.

- Acceso a agua potable en el área urbana del municipio de Intibucá

El área urbana del municipio está comprendida por la ciudad de Intibucá la que comprende 9 barrios y 2 colonias conformados por 2022 viviendas de las cuales 1867 (92.3\%) cuentan con agua de un sistema público o privado y 25 (1.2\%) viviendas que obtienen 
Cuadro No. 1: Procedencia del servicio de agua en las comunidades del municipio de Intibucá

\begin{tabular}{|c|c|c|c|c|c|c|c|c|c|}
\hline Aldeas & Comunidades & Viviendas & $\begin{array}{l}\text { Servicio } \\
\text { público } \\
\text { privado }\end{array}$ & $\begin{array}{c}\text { De } \\
\text { pozo o } \\
\text { malacate }\end{array}$ & $\begin{array}{c}\text { De pozo } \\
\text { con bom- } \\
\text { ba }\end{array}$ & $\begin{array}{l}\text { De ver- } \\
\text { tiente o } \\
\text { río }\end{array}$ & $\begin{array}{c}\text { De lago o } \\
\text { laguna }\end{array}$ & Comprada & Otros \\
\hline Azacualpa & 10 & 462 & 310 & 8 & 0 & 72 & 0 & 0 & 72 \\
\hline El Naranjo & 6 & 129 & 108 & 0 & 0 & 14 & 0 & 0 & 7 \\
\hline El Pelón de Ologosí & 6 & 209 & 75 & 1 & 3 & 118 & 4 & 0 & 8 \\
\hline Intibucá & $\begin{array}{c}2 \text { (12 barrios en } \\
\text { Intibucá) }\end{array}$ & 2073 & 1912 & 9 & 3 & 24 & 5 & 6 & 114 \\
\hline La Laguna de Chiligatoro & 7 & 243 & 171 & 4 & 1 & 51 & 3 & 0 & 13 \\
\hline La Sorto & 6 & 181 & 106 & 3 & 0 & 61 & 2 & 0 & 9 \\
\hline Malguara & 10 & 229 & 126 & 13 & 1 & 45 & 1 & 0 & 43 \\
\hline Manazapa & 4 & 137 & 84 & 26 & 0 & 18 & 0 & 0 & 9 \\
\hline Mixcure & 12 & 216 & 62 & 3 & 2 & 146 & 2 & 0 & 1 \\
\hline Monquecagua & 5 & 231 & 197 & 2 & 0 & 18 & 2 & 0 & 12 \\
\hline Pueblo Viejo & 4 & 186 & 114 & 18 & 1 & 39 & 0 & 0 & 14 \\
\hline Quebrada Honda & 5 & 242 & 194 & 9 & 0 & 30 & 0 & 0 & 9 \\
\hline Río Blanco & 11 & 238 & 139 & 0 & 0 & 41 & 0 & 0 & 58 \\
\hline Río Colorado & 8 & 136 & 63 & 17 & 0 & 49 & 0 & 0 & 7 \\
\hline Río Grande o El Nance & 3 & 176 & 142 & 6 & 0 & 26 & 0 & 0 & 2 \\
\hline San José & 6 & 198 & 98 & 2 & 0 & 95 & 0 & 0 & 3 \\
\hline San Nicolás & 6 & 248 & 93 & 0 & 0 & 149 & 1 & 0 & 5 \\
\hline San Pedro & 6 & 241 & 54 & 0 & 2 & 183 & 1 & 0 & 1 \\
\hline Santa Catarina & 7 & 278 & 203 & 1 & 2 & 62 & 0 & 0 & 10 \\
\hline Togopala & 5 & 205 & 37 & 0 & 0 & 165 & 0 & 0 & 3 \\
\hline TOTALES & 129 & 6,258 & 4,288 & 122 & 15 & 1,406 & 21 & 6 & 400 \\
\hline
\end{tabular}

Fuente: Elaboración propia según datos del CPV2001.

agua de un río o una laguna. El número de viviendas que no cuenta con un servicio de agua potable es bajo comparado con el de los que si tienen el servicio, sin embargo, es preocupante esta situación debido a que el agua que se consume en estas viviendas es el origen de enfermedades gastrointestinales, desnutrición y hasta la muerte de niños o niñas que por escasos recursos no logran controlarlas.

El gráfico No. 2 demuestra claramente que el mayor porcentaje de viviendas se concentra en la procedencia de agua de un sistema público o privado, podemos deducir que es un consumo de agua potable.

En el caso de las viviendas cuya fuente de agua procede de pozo de malacate y con bomba, el agua no es potable ya que el agua recibirá tratamiento sólo si las familias que la consumen implementan prácticas como
Gráfico 2: Número de viviendas según procedencia de agua, área urbana del municipio de Intibucá

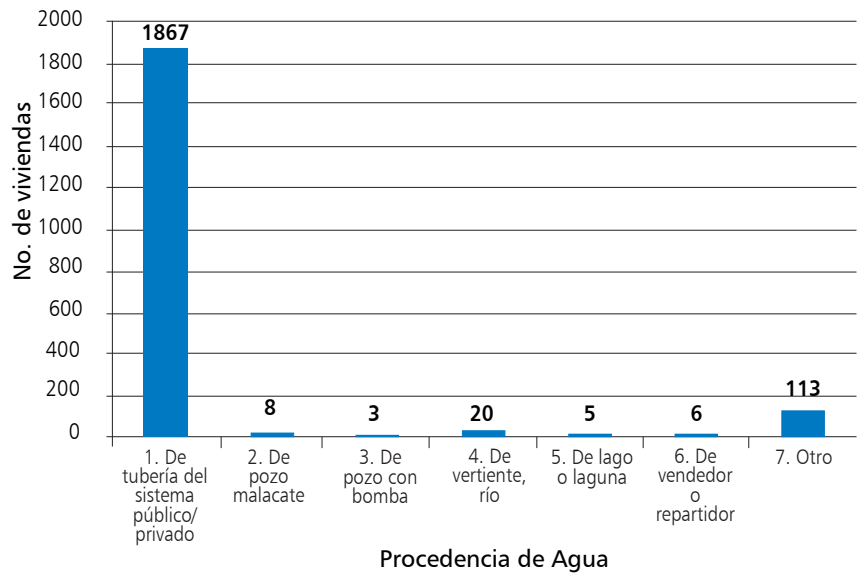

Fuente: Elaboración propia según datos del censo Poblacional y Vivienda del 2001.

hervirla antes de su consumo. Cabe mencionar que en estos sistemas no son muy cómodos para las mujeres, 
ya que se requiere de un esfuerzo mayor para obtener el agua, deben caminar, utilizar la fuerza para sacar el agua, realizar varios viajes para llevarla a casa, etc.

La procedencia de otros que aparece graficada representa un número de viviendas considerable que el censo contabiliza; pero son muchas veces encuestas que no logran realizarse por diferentes razones, principalmente por no encontrar informante en casa, cambios de domicilio, negativa a informar, etc. Lastimosamente para analizar si este número de viviendas cuenta con agua potable necesitamos de información actualizada y el nuevo censo es posible hasta 5 ó 10 años.

- Acceso a agua potable en el área rural del municipio de Intibucá

En el área rural contemplamos 4,236 viviendas de las cuales 2,421 (57.2\%) cuentan con servicio de agua potable y $1,381(32.6 \%)$ toman agua de un río o una laguna (Gráfico No. 3); esta situación preocupa más debido a que el financiamiento para proyectos de agua potable es más difícil de lograr por factores de accesibilidad que repercuten en el monto total del proyecto, el conformismo de la población afectada, el radio de acción de posibles entes financieros así como los insuficientes recursos económicos con que cuentan las municipalidades. Cabe recalcar que estas viviendas no están exentas de acceso a información sobre la importancia de prevenir enfermedades por consumir agua contamina, hoy en día, hay muchos avances de salud pública en cuanto a brindar la información de prevención así mismo los medios de comunicación accesibles a la zona son actores que también participan.

¿Cómo se logra el acceso al servicio de agua potable en el área rubal del municipio de Intibucá?

En el área rural la participación de entes externos ha sido muy importante para

\section{Gráfico No. 3. Número de viviendas según proceden-} cia de agua, área rural del municipio de Intibucá

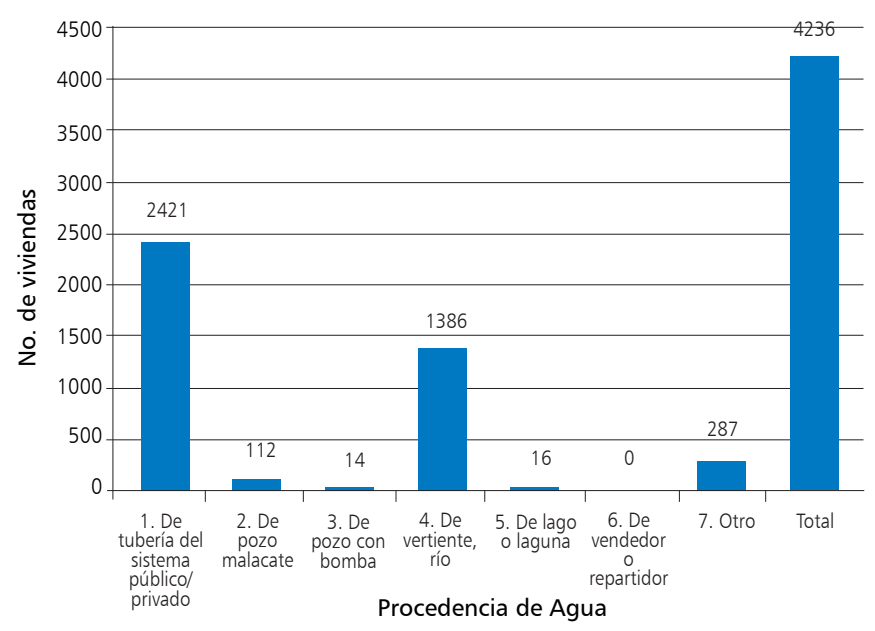

Fuente: Elaboración propia según datos del censo Poblacional y Vivienda del 2001.

ampliar y mejorar los servicios de agua potable, estos entes externos son ONG's presentes en el municipio de Intibucá.

El control de sistemas de agua potable en el área rural que registra el SANAA nos permite analizar el porcentaje de cobertura por cada una de las fuentes que han financiado proyectos de agua potable en el municipio, así mismo podemos destacar el porcentaje de participación de las instituciones que actualmente siguen encaminando esfuerzos para mejorar el sistema de agua potable en la zona

\section{Cuadro No. 2: Participación de instituciones en financiamiento de proyectos de agua potable del municipio de Intibucá}

\begin{tabular}{|l|l|l|l|c|c|}
\hline No. & $\begin{array}{c}\text { Institución } \\
\text { Financiadora }\end{array}$ & $\begin{array}{c}\text { No. } \\
\text { Proyectos }\end{array}$ & Población & $\begin{array}{c}\text { No. de } \\
\text { Viviendas }\end{array}$ & $\begin{array}{c}\text { No. De } \\
\text { Viviendas } \\
\text { beneficia- } \\
\text { rias }\end{array}$ \\
\hline 1 & Save The Childrens & 19 & 15,836 & 1,813 & 1,542 \\
\hline 2 & Gobierno: Salud Publica & 6 & 3,034 & 568 & 221 \\
\hline 3 & COCEPRADI & 5 & 2,388 & 380 & 327 \\
\hline 4 & Visión Mundial & 3 & 2,756 & 460 & 343 \\
\hline 5 & CRS & 3 & 770 & 120 & 94 \\
\hline 6 & SANAA-UNICEF & 2 & 546 & 91 & 70 \\
\hline 7 & SANAA-CARE & 1 & 408 & 68 & 68 \\
\hline 8 & SANAA-AID & 1 & 894 & 149 & 118 \\
\hline 9 & FEDECO y EDUCSA & 1 & 300 & 50 & 42 \\
\hline \multicolumn{7}{|l|}{ TOTAL } & 41 & 26,932 & 3,699 & 2,825 \\
\hline Cobertura & & & & $76.37 \%$ \\
\hline
\end{tabular}

Fuente: Elaboración propia según datos Sistema de Instalaciones de Acueductos Rurales. (SIAR CTOCC, 05-ago-2008) 
rural del municipio, tal es el caso de Save The Children que ha financiado el $43.3 \%$ de los proyectos, entidades gubernamentales como Salud Pública y el IHNFA con un 14.6\% y Visión Mundial un 7.3\%. Cabe destacar que el SANAA como contraparte de otras fuentes ha logrado una participación de $9.8 \%$ y es la institución que activamente se encuentra monitoreando los sistemas de agua establecidos. El Gráfico No. 4 refleja el porcentaje de cobertura considerando el número de viviendas total de cada proyecto vrs el número de viviendas beneficiarias, también se logra comparar el número de proyectos en los que cada fuente financiadora ha intervenido.

En la relación SANAA-CARE se presenta un porcentaje de cobertura del $100 \%$ pero en un solo proyecto de agua; el cubrir el número total de viviendas de una comunidad es lo ideal en cualquier proyecto no obstante, siempre se presentan limitantes para lograrlo, esta aseveración podría justificar el porcentaje logrado en las otras fuentes de financiamiento. La situación de Save The Children es importante resaltar que no presenta el porcentaje de cobertura más alto pero si mayor número de proyectos de agua financiados, indudablemente se refleja un alto compromiso de esta $\mathrm{ONG}$ en mejorar las condiciones de vida de la población que atienden.

\section{Gráfico 4: Porcentaje de cobertura de fuentes financiadoras de proyectos de agua potable en el municipio de Intibucá}

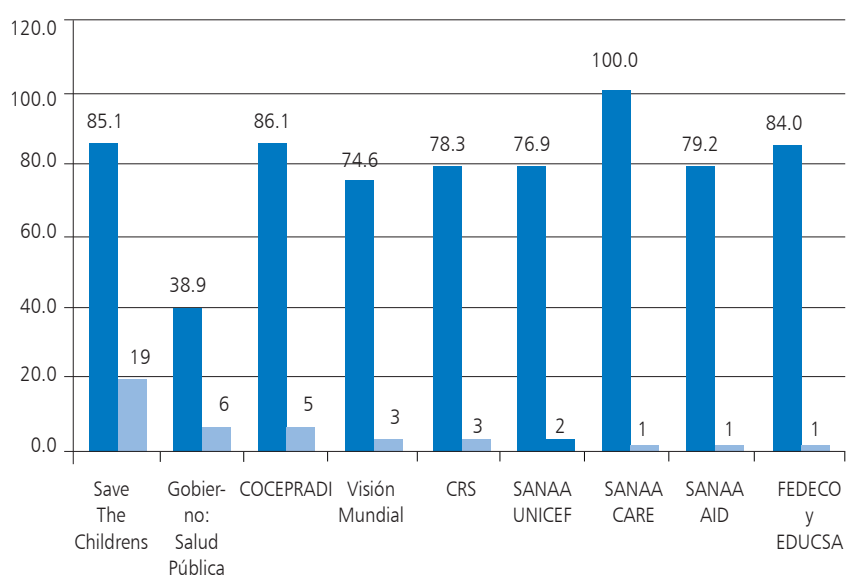

Fuente: Elaboración propia según datos Sistema de Instalaciones de Acueductos Rurales. (SIAR CTOCC, 05-ago-2008)
El cuadro No. 3 presenta el número de viviendas dentro de cada comunidad que han sido beneficiadas con un proyecto de agua potable, y son proyectos que reciben asistencia técnica de personal del SANAA. Como ya se ha mencionado el SANAA establece alianzas con ONGs para financiar estos proyectos en el área rural. Todas las actividades de mantenimiento del sistema están a cargo de las Juntas de Agua quienes establecen planificaciones mensuales de actividades de supervisión de la fuente y de aseo de las represas y tanques.

La población atendida asciende a 26,932 personas ubicadas en 3,699 viviendas de las cuales el 76.4\% cuenta ya con el servicio de agua potable, el municipio de Intibucá está conformado por 129 comunidades y el SANAA tiene influencia en la actualidad en 41 comunidades lo que representa un 31.8\%. El censo del 2001 contabiliza 6258 viviendas de las cuales sólo 4288 contaban con servicio de agua potable en la información del SIAR del SANAA hasta agosto del 2008 se atendieron a 2825 viviendas lastimosamente no podríamos decir que son viviendas diferentes o iguales a las del censo. Si son nuevas podríamos decir que se ha disminuido la cantidad de viviendas sin agua, pero esto es un supuesto, así mismo se debe tomar en cuenta que para este año 2008 ha aumentado la población y por ende la cantidad de viviendas especialmente en el área rural.

\section{- Potencialidades del servicio que presta el SANAA}

Los sistemas rurales construidos por el SANAA incorporan una importante participación comunitaria y su gestión se delega a juntas administradoras integradas por usuarios. Los sistemas urbanos menores y los rurales construidos por otras dependencias oficiales y organizaciones no gubernamentales siguen prácticas similares y la operación recae en las municipalidades, patronatos o juntas administradoras.

El servicio que presta el SANAA es meritorio, el registro de información que están actualizando según la ejecución de proyectos de agua potable, es una herramienta que facilitará análisis más certeros con la información que brinde el próximo censo de población 
Cuadro No. 3. Número de viviendas con servicio de agua potable en las comunidades del municipio de Intibucá asistidas por el SANAA

\begin{tabular}{|c|c|c|c|c|c|}
\hline No. & Comunidad & Población & Viviendas & Clientes & Cobertura \\
\hline 1 & Azacualpa & 4320 & 72 & 72 & 1 \\
\hline 2 & Azacualpa \#2 & 1296 & 217 & 100 & 0.460829493 \\
\hline 3 & El Tabor Azacualpa & 408 & 68 & 68 & 1 \\
\hline 4 & Goangololo Rev Azacualpa & 1278 & 213 & 213 & 1 \\
\hline 5 & Quiaterique Azacualpa & 530 & 90 & 72 & 0.8 \\
\hline 6 & Cedros Rv San José & 150 & 35 & 26 & 0.742857143 \\
\hline 7 & San José & 750 & 65 & 65 & 1 \\
\hline 8 & Centro Miscure & 250 & 37 & 25 & 0.675675676 \\
\hline 9 & Mixcure & 210 & 35 & 24 & 0.69 \\
\hline 10 & Palmas Mixcure & 224 & 37 & 34 & 0.92 \\
\hline 11 & Chiligatoro, Pueblo Viejo \#2, Palistal & 320 & 60 & 45 & 0.75 \\
\hline 12 & El Cacao Quebrada Honda & 650 & 108 & 108 & 1 \\
\hline 13 & Los Encinos Quebrada Honda & 432 & 72 & 60 & 0.833333333 \\
\hline 14 & El Cipres Rev Monquecagua & 372 & 62 & 42 & 0.677419355 \\
\hline 15 & Monquecagua & 1260 & 200 & 170 & 0.85 \\
\hline 16 & El Molino De Intibucá & 360 & 60 & 39 & 0.65 \\
\hline 17 & Llano de La Virgen & 1836 & 306 & 306 & 1 \\
\hline 18 & Quebrada de Lajas & 630 & 65 & 50 & 0.769230769 \\
\hline 19 & El Rosario Revisar en San Nicolás & 226 & 35 & 33 & 0.94 \\
\hline 20 & San Antonio Revisar San Nicolás & 186 & 31 & 31 & 1 \\
\hline 21 & San Nicolás & 400 & 130 & 40 & 0.307692308 \\
\hline 22 & El Zapote Revi Togopala & 348 & 58 & 38 & 0.655172414 \\
\hline 23 & Togopala & 810 & 135 & 135 & 1 \\
\hline 24 & Guachipilincito Revisa Río Blanco & 88 & 14 & 13 & 0.93 \\
\hline 25 & La Tejera Río Blanco & 750 & 125 & 55 & 0.44 \\
\hline 26 & Valle de Ageles Río Blanco & 434 & 59 & 47 & 0.8 \\
\hline 27 & Las Mercedez, Manazapa & 240 & 40 & 40 & 1 \\
\hline 28 & Manazapa & 408 & 68 & 48 & 0.705882353 \\
\hline 29 & Malguara & 1254 & 209 & 85 & 0.406698565 \\
\hline 30 & Pelon Ologosi & 480 & 80 & 60 & 0.75 \\
\hline 31 & Peña Blanca Rev El Naranjo & 354 & 59 & 43 & 0.73 \\
\hline 32 & Pinares-Terreros Revisa Pueblo Viejo & 1044 & 174 & 125 & 0.718390805 \\
\hline 33 & Pueblo Viejo \#1 & 730 & 37 & 29 & 0.783783784 \\
\hline 34 & Planes Río Grande & 894 & 149 & 118 & 0.79 \\
\hline 35 & Río Grande & 600 & 100 & 70 & 0.7 \\
\hline 36 & Quebrada de Vuelta Rev Sorto & 300 & 50 & 42 & 0.84 \\
\hline 37 & Río Colorado & 522 & 87 & 57 & 0.66 \\
\hline 38 & San Pedro El Duraznito & 468 & 78 & 58 & 0.743589744 \\
\hline 39 & San Pedro La Loma Silimania & 606 & 101 & 70 & 0.69 \\
\hline 40 & Santa Catarina & 328 & 47 & 38 & 0.81 \\
\hline \multirow[t]{3}{*}{41} & Villa Francis Sta. Catarina & 186 & 31 & 31 & 1 \\
\hline & Total & 26,932 & 3699 & 2825 & \\
\hline & Porcentaje De Cobertura & \multicolumn{4}{|c|}{$76.4 \%$} \\
\hline
\end{tabular}

Fuente: Elaboración propia según datos Sistema de Instalaciones de Acueductos Rurales. (SIAR CTOCC, 05-ago-2008) 
y vivienda del país. El mérito también se lo merece por el esfuerzo concreto en lograr que la población cuente con el derecho humano agua.

El SANAA funciona como una entidad de apoyo técnico, encargado del desarrollo de la cobertura rural. Los sistemas rurales requieren una labor de supervisión y apoyo permanente, las cuales se brindan mediante los Técnicos en Operación y Mantenimiento (TOM) cuya remuneración y dotación de recursos ha recaído en los proyectos de inversión.

- Limitaciones del servicio prestado por SANAA:

Una vez finalizados los proyectos de agua rural, la asistencia técnica brindada por los TOM es incierta y así mismo la participación de OPD's de apoyar a la comunidad en estas intervenciones, también harán falta recursos de capital para rehabilitación, ampliación y mejoras. Esta deberá ser una responsabilidad de gestión del gobierno local y gobierno nacional.

A pesar de los esfuerzos de que toda la población urbana y rural cuente con el servicio de agua potable, todavía es una meta que no se logra alcanzar en su totalidad. El fenómeno en el área rural es que la mayoría de viviendas cuenta con la conexión del servicio pero la cantidad de tiempo y volumen de agua es limitado. Según empleados del SANAA, la capacidad de las fuentes utilizadas no es suficiente ya que la red de distribución ha aumentado considerablemente, así mismo trabajos de mejoramiento en el alcantarillado no permiten un fluido continuo del agua en la red. Se espera que sea una realidad el proyecto de canalización del agua que vierte en los baños públicos para aumentar el volumen en la red de distribución actual del área urbana de La Esperanza y de Intibucá.

\section{CONCLUSIONES}

1. Según los datos del CPV 2001 sólo el $68.5 \%$ de las viviendas del municipio de Intibucá cuenta con un servicio de agua potable, servicio que es público y en algunos casos privados por un financiamiento compartido entre el gobierno y un patronato o junta de agua. Un 22.5\% no cuenta con un sistema de agua en condiciones saludables para las personas, de este $22.5 \%$ el $90 \%$ obtiene el agua de una quebrada o río convirtiéndose en un número de viviendas de 1,406 donde sus habitantes están expuestos a múltiples enfermedades gastrointestinales.

2. El área rural del municipio de Intibucá refleja los porcentajes mayores en número de viviendas que no cuentan con el servicio de agua potable. El área rural comprende el $67.7 \%(4,236)$ del total de viviendas del municipio de Intibucá, del cual 57.2\% cuenta con servicio de agua potable y el $32.6 \%$ toma agua de un río o una laguna; esto no demuestra que el no contar con agua potable es una necesidad que se agudiza más en el área rural que en el área urbana como es la generalidad de

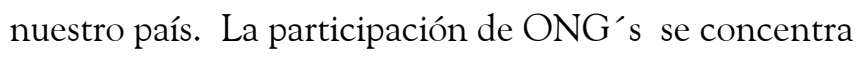
en el área rural por lo tanto son la oportunidad esperanzadora de solventar la necesidad básica de agua en las comunidades más afectadas.

3. Es notable que lograr la cobertura total de viviendas con acceso a agua potable no es posible sin el compromiso de los afectados, ONG's y gobiernos locales. En el área rural del municipio de Intibucá las ONG’s han cumplido y cumplen un papel importante para lograr metas en cuanto al mejoramiento de la calidad de vida de las familias. Un financiamiento compartido entre el gobierno y un sector privado facilita el cumplimiento de metas, y si a eso agregamos la contraparte de participación de las familias beneficiarias el resultado será un proyecto exitoso y con compromiso de mantenimiento.

4. El sub registro de información en instituciones nacionales se presenta como una desventaja para analizar cantidades considerables de viviendas con acceso al agua. Hay muchas disparidades de cantidades que se presentan en el CPV 2001 en comparación con información actualizada al 2008 que registra el Servicio de Información de Sistemas Rurales del SANAA. (SIAR CTOCC, 05-ago-2008). Son notables las diferencias en el número de viviendas atendidas vrs el número total de viviendas contabilizadas en el censo. 


\section{BIBLIOGRAFÍA}

- $\quad$ (C) 1997 Monografias.com S.A. "Derecho Humano al agua Potable". Sin autor.

- Copyright $₫ 2008$, Programa de las Naciones Unidas para el Desarrollo.

- Diario el TIEMPO, Sección: INFORME. Desarrollo Humano, Agua y saneamiento, el desafío del gobierno. Jueves 17 de Julio de 2008.

- Jouravlev Andrei, Los servicios de agua potable y saneamiento en el umbral del siglo XXI.
- INE, Anuario Estadístico 2006. Aspectos Demográficos paginas 77-80.

- INE, Anuario Estadístico 2006. Aspectos Sociales, Salud, paginas $187-189$.

- INE 2001, XVI Censo de Poblacional y Vivienda, 2001

- Hoja de calculo EXCEL, Sistema de Instalaciones de Acueductos Rurales (SIAR CTOCC, 05-ago-2008), SANAA, Siguatepeque.

- Wikipedia.org, Agua potable y Saneamiento en Honduras.

- $\quad$ www.monografias.com

\section{ANEXOS}

Base de datos del Sistema de Instalaciones de Acueductos Rurales (SIAR CTOCC, 5-ago-08)

\begin{tabular}{|c|c|c|c|c|c|c|c|c|c|c|c|}
\hline \begin{tabular}{|l|} 
Comunidad \\
\end{tabular} & POBLACIC & NVIVIENDAS & S SER VPUB 1 & CLIENTES & COBERTUR & FINANCIA & TARIF & ATIPOSISTE & TIPOFUENT & ENOMBRFTES 1 & QFUENTE 1 \\
\hline $\begin{array}{ll}\text { EL } & \text { CACAO } \\
\text { QUEBRADA HONDA }\end{array}$ & 650 & 108 & ESCUELA, IGLESIA & 108 & 1 & VISION MUNDIAL & 10 & Gravedad & NACIMIENTO & PUEBLO VIEJO & 19,6 GPM \\
\hline \begin{tabular}{|lr} 
LOS & ENCINOS \\
QUEBRADA HONDA
\end{tabular} & 432 & 72 & ESCUELA,IGLESIAS & 60 & 0.833333333 & $\begin{array}{ll}\begin{array}{l}\text { SAVE } \\
\text { CHILDREN }\end{array} & \text { THE } \\
\end{array}$ & 10 & Gravedad & NACIMIENTO & MANAZAPA & $55.7 \mathrm{GPM}$ \\
\hline & 1082 & 180 & 0 & 168 & & & & & & & \\
\hline \begin{tabular}{|lcl} 
EL & CIPRES & REV \\
MONQUECAGUA
\end{tabular} & 372 & 62 & NINGUNO & 42 & 0.677419355 & $\begin{array}{ll}\begin{array}{l}\text { SAVE } \\
\text { CHELDREN }\end{array} & \text { THE } \\
\end{array}$ & 8 & Gravedad & NACIMIENTO & ALTOS DEL CIPRES & $18 \mathrm{GPM}$ \\
\hline MONQUECAGUA & 1260 & 200 & \begin{tabular}{|l|} 
ESCUELA,IGLESIA, \\
CENTRO DE SALUD, \\
COLEGIO \\
\end{tabular} & 170 & 0.85 & \begin{tabular}{|lr} 
SAVE & THE \\
CHILDREN & \\
\end{tabular} & 6 & Gravedad & NACIMIENTO & & 12 GPM \\
\hline & 1632 & 262 & \begin{tabular}{l|l|}
0 \\
\end{tabular} & 212 & & & & & & & \\
\hline $\begin{array}{|lrl|}\text { EL } & \text { MOLINO } & \text { DE } \\
\text { INTIBUCA }\end{array}$ & 360 & 60 & \begin{tabular}{|lr}
$\begin{array}{l}\text { ESCUELA, } \\
\text { KINDER }\end{array}$ \\
\end{tabular} & 39 & 0.65 & SANAA UNICEF & 24 & Gravedad & NACIMIENTO & MARASIAS & 22.00 \\
\hline \begin{tabular}{|lll} 
LLANO & DE & LA \\
VIRGEN & & \\
\end{tabular} & 1836 & 306 & $\begin{array}{l}\text { ESCUELA,ENEE,TELE } \\
\text { FONO }\end{array}$ & 306 & 1 & $\begin{array}{ll}\begin{array}{l}\text { SAVE } \\
\text { CHILDREN }\end{array} & \text { THE } \\
\end{array}$ & 10 & Gravedad & NACIMIENTO & EL PAISLAL & 28 G.P.M \\
\hline $\begin{array}{l}\text { QUEBRADA } \\
\text { LAJAS } \\
\end{array}$ & 630 & 65 & \begin{tabular}{|l|} 
ESCUELA,IGLESIA, \\
SAVE \\
CHILDRENS THE \\
\end{tabular} & 50 & 0.769230769 & SAVE CHILDREN & 10 & Gravedad & NACIMIENTO & EL PASTAL & 15 GPM \\
\hline & 2826 & 431 & 0 & 395 & & & & & & & \\
\hline \begin{tabular}{|lll} 
EL & ROSARIO \\
REVISAR & EN & SAN \\
NICOLAS & & \\
\end{tabular} & 226 & 35 & ESCUELA IGLESIA & 33 & 0.94 & \begin{tabular}{|lr} 
JUNTA & DE \\
BIENESTAR SOCIAL
\end{tabular} & 10 & Gravedad & NACIMIENTO & EL MANGO & 9 G.P.M. \\
\hline $\begin{array}{|lr|}\text { SAN } & \text { ANTONIO } \\
\text { REVISAR } & \text { SAN } \\
\text { NICOLAS } & \\
\end{array}$ & 186 & 31 & ESCUELA, IGLESIA. & 31 & 1 & SANAA-UNICEF & 70 & Mixto (G/B) & NACIMIENTO & $\begin{array}{l}\text { "EL SAPOTE' } \\
\text { "LA BOMBA" }\end{array}$ & $9.6 \mathrm{GPM}$ \\
\hline SAN NICOLAS & 400 & 130 & $\begin{array}{|lr|}\text { ESCUELA, } & \text { CENTRO } \\
\text { DE SALUD, IGLESIA, } \\
\text { COLEGIO, KINDER } \\
\end{array}$ & 40 & 0.307692308 & GOBIERNO & 5 & Gravedad & NACIMIENTO & CERRO VERDE & 60 GPM \\
\hline & 812 & 196 & 0 & 104 & & & & & & & \\
\hline $\begin{array}{lll}\text { EL } & \text { ZAPOTE } \\
\text { TOGOPALA } & \text { REVI } \\
\end{array}$ & 348 & 58 & \begin{tabular}{|l|} 
ESCUELAS-IGLESIA \\
\end{tabular} & 38 & 0.655172414 & $\begin{array}{l}\text { SAVE } \\
\text { CHILDREN }\end{array}$ & 10 & Gravedad & NACIMIENTO & EL ZAPOTE & \begin{tabular}{|lrl} 
NO & SE & PUDO \\
AFORAR & \\
\end{tabular} \\
\hline
\end{tabular}


Base de datos del Sistema de Instalaciones de Acueductos Rurales (SIAR CTOCC, 5-ago-08)

\begin{tabular}{|c|c|c|c|c|c|c|c|c|c|c|c|}
\hline Comunidad & POBLACIO & NVIVIENDAS & SERVPUB 1 & CLIENTES & COBERTURA & FIN ANCIA & TARIFA & ATIPOSISTE & TIPOFUENTE & NOMBRFTES 1 & QFUENTE 1 \\
\hline AZACUALPA & 4320 & 72 & \begin{tabular}{|l} 
ESCUELA \\
ESCUELA
\end{tabular} & 72 & 1 & $\begin{array}{|ll|}\text { SAVE } & \text { THE } \\
\text { CHILDRENS } & \\
\end{array}$ & 6 & Gravedad & NACIMIENTO & \begin{tabular}{|l|} 
LAS PAVAS \\
\end{tabular} & 16,66 GPM \\
\hline AZACUALPA \#2 & 1296 & 217 & $\begin{array}{l}\text { COLEGIO, ESCUELA, } \\
\text { IGLESIA }\end{array}$ & 100 & 0.460829493 & $\begin{array}{lrr}\text { VISION } & \text { MUNDIAL, } \\
\text { PDA, } & \text { SABE } & \text { THE } \\
\text { CHILDRENS } & \end{array}$ & 10 & Gravedad & NACIMIENTO & LAS DITENCIAS & 78,40 GPM \\
\hline $\begin{array}{|ll|}\text { EL } & \text { TABOR } \\
\text { AZACUALPA } & \\
\end{array}$ & 408 & 68 & ESCUELA-IGLESIA & 68 & 1 & SANAA-CARE & 5 & Gravedad & NACIMIENTO & EL LEON & 50 G.P.M \\
\hline $\begin{array}{|ll|}\text { GOANGOLOLO REV } \\
\text { AZACUALPA }\end{array}$ & 1278 & 213 & $\begin{array}{l}\text { ESCUELA,IGLESIA,CE } \\
\text { NTRO COMUNAL }\end{array}$ & 213 & 1 & $\begin{array}{|ll|}\begin{array}{l}\text { SAVE } \\
\text { CHILDREN }\end{array} & \text { THE } \\
\end{array}$ & 5 & Gravedad & NACIMIENTO & LOS OLIVOS & 74.,4 G.P.M \\
\hline $\begin{array}{l}\text { QUIATERIQUE } \\
\text { AZACUALPA } \\
\end{array}$ & 530 & 90 & $\begin{array}{l}\text { ESCUELA,CENTRO } \\
\text { DE SALUD, IGLESIA, } \\
\text { OPD,S }\end{array}$ & 72 & 0.8 & SALUD PUBLICA & 10 & Gravedad & NACIMIENTO & QUIATERIQUE & 35 GPM \\
\hline & 7832 & 660 & & 525 & & & & & & & \\
\hline $\begin{array}{|lll|}\text { CEDROS } & \text { RV } & \text { SAN } \\
\text { JOSE } & & \\
\end{array}$ & 150 & 35 & ESCUELA,IGLESIAS & 26 & 0.742857143 & $\begin{array}{|ll|}\text { SAVE } & \text { THE } \\
\text { CHELDREN } & \\
\end{array}$ & 10 & Gravedad & NACIMIENTO & LA CAJA & 10 GPM \\
\hline SAN JOSE & 750 & 65 & ESCUELA IGLESIAS & 65 & 1 & SAVE CHILDREN & 10 & Gravedad & NACIMIENTO & SAN JOSE & 40 \\
\hline & 900 & 100 & 0 & 91 & & & & & & & \\
\hline CENTRO MISCURE & 250 & 37 & ESCUELA, IGLESIA & 25 & 0.675675676 & COCEPRADII & 5 & Gravedad & NACIMIENTO & EL CERRON & $60 \mathrm{GPM}$ \\
\hline MIXCURE & 210 & 35 & ESCUELA,IGLESIAS & 24 & 0.69 & COCEPRADII & 5 & Gravedad & QUEBRADA & $\begin{array}{|lc|}\text { 1.LA } & \text { CUMBRE } \\
\text { 2.LOS OLIVOS }\end{array}$ & $\begin{array}{l}\text { 1- } 30 \text { GPM, 2- } 76 \\
\text { GPM }\end{array}$ \\
\hline PALMAS MIXCURE & 224 & 37 & ESCUELA,IGLESIAS & 34 & 0.92 & \begin{tabular}{|l|} 
SAVE \\
CHELDREN \\
\end{tabular} & 4 & Gravedad & NACIMIENTO & \begin{tabular}{|l|l|} 
LAS PALMAS \\
\end{tabular} & 24.6 G.PM \\
\hline & 684 & 109 & 0 & 83 & & & & & & & \\
\hline \begin{tabular}{|l|} 
CHILIGATORO, \\
PUEBLO VIEJO \#2, \\
PALISTAL \\
\end{tabular} & 320 & 60 & $\begin{array}{l}\text { ESCUELA,IGLESIA, } \\
\text { OPD,S. }\end{array}$ & 45 & 0.75 & $\begin{array}{l}\text { SAVE } \\
\text { CHILDREN }\end{array}$ & 15 & Gravedad & NACIMIENTO & LA MICRO CUENCA & 90 GPM \\
\hline & 320 & 60 & 0 & 45 & & & & & & & \\
\hline
\end{tabular}

Base de datos del Sistema de Instalaciones de Acueductos Rurales (SIAR CTOCC, 5-ago-08)

\begin{tabular}{|c|c|c|c|c|c|c|c|c|c|c|c|}
\hline Comunidad & POBLACIO & NVIVIENDAS & SER VPUB 1 & CLIENTES & COBERTURA & FINANCIA & TARIFA & ATIPOSISTE & TIPOFUENTE & NOMBRFTES 1 & QFUENTE 1 \\
\hline TOGOPALA & 810 & 135 & ESCUELA,IGLESIAS & 135 & 1 & $\begin{array}{l}\text { P.D.A.(VISION } \\
\text { MUNDIAL) }\end{array}$ & 10 & Gravedad & NACIMIENTO & \begin{tabular}{|l|} 
GUANSAUCE \\
\end{tabular} & 75.7 GPM \\
\hline & $\#$ \#EF! & $\#$ \#RE! & \#iREF! & \#iREF! & & & & & & & \\
\hline $\begin{array}{l}\text { GUACHIPILINCITO } \\
\text { REVISA RIO BLANCO }\end{array}$ & 88 & 14 & ESCUELA IGLESIA & 13 & 0.93 & CRS & 5 & Gravedad & NACIMIENTO & GUACHIPILIN & 6.2 G.P.M \\
\hline 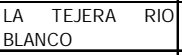 & 750 & 125 & \begin{tabular}{|l} 
ESCUELA,CENTRO \\
DE SALUD,IGLESIA \\
\end{tabular} & 55 & 0.44 & $\begin{array}{l}\text { SALUD,CUERPO DE } \\
\text { PAZ }\end{array}$ & 0 & Gravedad & NACIMIENTO & EL GUEGUECHO & $45 \mathrm{G} / \mathrm{M}$ \\
\hline $\begin{array}{l}\text { VALLE DE AGELES } \\
\text { Rio Blanco } \\
\end{array}$ & 434 & 59 & \begin{tabular}{|l|} 
ESCUELA,CENTRO \\
DE SALUD,IGLESIA \\
\end{tabular} & 47 & 0.8 & COSEPRADII & 3 & Gravedad & NACIMIENTO & EL LIQUIDANBO & $125 \mathrm{G} / \mathrm{M}$ \\
\hline & 1272 & 198 & 0 & 115 & & & & & & & \\
\hline 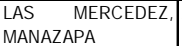 & 240 & 40 & ESCUELA, IGLESIA & 40 & 1 & COCEPREDII & 10 & Gravedad & NACIMIENTO & LOS LIQUIDAMBOS & 120 GPM \\
\hline \begin{tabular}{|l|l|} 
MANAZAPA \\
\end{tabular} & 408 & 68 & ESCUELA, IGLESIA & 48 & 0.705882353 & $\begin{array}{|lr|}\text { SABE } & \text { THE } \\
\text { CHILDREN, CARE }\end{array}$ & 10 & Gravedad & NACIMIENTO & CERRO SAN JUAN & $90 \mathrm{GPM}$ \\
\hline & 648 & 108 & 0 & 88 & & & & & & & \\
\hline MALGUARA & 1254 & 209 & $\begin{array}{l}\text { ESCUELA,IGLESIA, } \\
\text { COLEGIO } \\
\end{array}$ & 85 & 0.406698565 & COSEPRADI & 8 & Gravedad & NACIMIENTO & EL SIPRES & 32 GPM \\
\hline & 1254 & 209 & 0 & 85 & & & & & & & \\
\hline PELON OLOGOSI & 480 & 80 & ESCUELA & 60 & 0.75 & $\begin{array}{|ll|}\text { SAVE } & \text { THE } \\
\text { CHILDREN } & \\
\end{array}$ & 10 & Gravedad & NACIMIENTO & MANGUARE & 27 G.P.M \\
\hline & 480 & 80 & 0 & 60 & & & & & & & \\
\hline $\begin{array}{l}\text { PEÑA BLANCA REV } \\
\text { EL NARANJO } \\
\end{array}$ & 354 & 59 & IGLESIAS & 43 & 0.73 & C.R.S. COCEPRADII & 6 & Gravedad & NACIMIENTO & LA PEÑA & 28.6 G.P.M \\
\hline & 354 & 59 & 0 & 43 & & & & & & & \\
\hline \begin{tabular}{|l|} 
PINARES- \\
TERREROS REVISA \\
PUEBLO VIEJO \\
\end{tabular} & 1044 & 174 & ESCUELA,IGLESIA & 125 & 0.718390805 & $\begin{array}{|ll|}\text { SANAA PRASAR- } & \text { PAV } \\
\text { SAVE THE CHIL }\end{array}$ & 10 & Gravedad & NACIMIENTO & $\begin{array}{|ll|}\text { PUEBLO VIEJO (18 } \\
\text { GLS), Y } & \text { PINIARES } \\
(24 \text { GLS) } & \\
\end{array}$ & \begin{tabular}{|lll}
42 & GLS & POR \\
MIN. & & \\
\end{tabular} \\
\hline \begin{tabular}{|l|} 
PUEBLO VIEJO \#1 \\
\end{tabular} & 730 & 37 & $\begin{array}{l}\text { ESCUELA-IGLESIA, } \\
\text { OPD,S. }\end{array}$ & 29 & 0.783783784 & $\begin{array}{|ll|}\begin{array}{l}\text { SAVE } \\
\text { CHILDREN }\end{array} & \text { THE } \\
\end{array}$ & 15 & Gravedad & NACIMIENTO & CRUZ ALTA & 12 GPM \\
\hline & 1774 & 211 & 0 & 154 & & & & & & & \\
\hline
\end{tabular}

\begin{tabular}{|c|c|c|c|c|c|c|c|c|c|c|c|}
\hline & & ase de dat & tos del S istema & de Instala & aciones d & Acueductos Ru & ral & SIAR C & CC, 5-ago & & \\
\hline $\begin{array}{l}\text { PLANES } \\
\text { GRANDE }\end{array}$ & 894 & 149 & \begin{tabular}{|l|} 
ESCUELA,IGLESIAS,C \\
ENTRO DE SALUD
\end{tabular} & 118 & 0.79 & SANAA-AID & 10 & Gravedad & NACIMIENTO & $\begin{array}{l}\text { NO TIENE(HAY } \\
\text { FUENTES) }\end{array}$ & 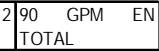 \\
\hline RIO GRANDE & 600 & 100 & $\begin{array}{|lr|}\text { ESCUELA, IGLESIA, } \\
\text { CENTRO DE SALUD } \\
\end{array}$ & 70 & 0.7 & \begin{tabular}{|lc} 
SAVE & THE \\
CHILDREN & \\
\end{tabular} & 10 & Gravedad & NACIMIENTO & MANAZAPA & 58.6 GPM \\
\hline & 1494 & 249 & 0 & 188 & & & & & & & \\
\hline QUEBRADA DE & 300 & 50 & ESCUELA, IGLESIA & 42 & 0.84 & FEDECO Y EDUCSA & 4 & Gravedad & NACIMIENTO & SIMON CABRERA & 30 GPM \\
\hline VUELTA REV SORTO & & & & & & & & & & & \\
\hline & 300 & 50 & 0 & 42 & & & & & & & \\
\hline RIO COLORADO & 522 & 87 & ESCUELA-IGLESIA & 57 & 0.66 & SALUD PUBLICA & 8 & Gravedad & $\mathrm{RIO}$ & RIO SAN MIGUEL & 76 G.P.M \\
\hline & 522 & 87 & 0 & 57 & & & & & & & \\
\hline $\begin{array}{|lrl|}\text { SAN } & \text { PEDRO } & \text { EL } \\
\text { DURAZNITO } & \\
\end{array}$ & 468 & 78 & $\begin{array}{l}\text { ESCUELA, CENTRO, } \\
\text { IGLESIA }\end{array}$ & 58 & 0.743589744 & \begin{tabular}{|ll} 
SAVE & THE \\
CHILDREN & \\
\end{tabular} & 8 & Gravedad & NACIMIENTO & AGUA LINDA & 24.5 GPM \\
\hline $\begin{array}{|lcc|}\text { SAN } & \text { PEDRO } & \text { LA } \\
\text { LOMA } & \text { SILIMANIA } \\
\end{array}$ & 606 & 101 & \begin{tabular}{|l|} 
ESCUELA, \\
IGLESIA,OPD,S \\
\end{tabular} & 70 & 0.69 & SALUD PUBLICA & 5 & Gravedad & NACIMIENTO & GUASCOTORO & 15 GPM \\
\hline & 1074 & 179 & 0 & 128 & & & & & & & \\
\hline SANTA CATARINA & 328 & 47 & ESCUELA & 38 & 0.81 & CRS & 3 & Gravedad & NACIMIENTO & EL CEIBO & 30 G.P.M. \\
\hline \begin{tabular}{|l|} 
VILLA FRANCIS Sta \\
CATARINA \\
\end{tabular} & 186 & 31 & \begin{tabular}{|l|} 
IGLESIA,JARDIN DE \\
NINNOS
\end{tabular} & 31 & 1 & \begin{tabular}{|l|} 
SAVE \\
CHILDREN \\
\end{tabular} & 15 & Gravedad & NACIMIENTO & MARACILLA & $\begin{array}{|lr|}12 & \text { GALONES } \\
\text { POR MIN. }\end{array}$ \\
\hline & 514 & 78 & 0 & 69 & & & & & & & \\
\hline & & & & & & & & & & & \\
\hline
\end{tabular}

